\title{
PROBABILITY METHODS IN SOME PROBLEMS OF ANALYSIS AND NUMBER THEORY
}

\author{
M. KAC
}

1. Introduction. In 1922 Rademacher [ 1$]^{1}$ introduced the functions

$$
r_{n}(t)=\operatorname{sign} \sin 2^{n} \pi t, \quad 0 \leqq t \leqq 1, n=1,2, \cdots,
$$

and proved that the series

$$
\sum_{1}^{\infty} c_{n} r_{n}(t)
$$

converges almost everywhere provided

$$
\sum_{1}^{\infty} c_{n}^{2}<\infty
$$

In 1925 Kolmogoroff and Khintchine [1] generalized this result and also proved the counterpart to the effect that

$$
\sum_{1}^{\infty} c_{n}^{2}=\infty
$$

implies divergence almost everywhere of (1.2). The probabilistic nature of these results (first recognized by Steinhaus [1]) becomes apparent when one notices that the Rademacher functions $r_{n}(t)$ are statistically independent, that is, have the property that

$$
\text { (1.5) }\left|\underset{t}{E}\left\{r_{1}(t)<\alpha_{1}, \cdots, r_{n}(t)<\alpha_{n}\right\}\right|=\prod_{1}^{n}\left|\underset{t}{E}\left\{r_{k}(t)<\alpha_{k}\right\}\right|, 2
$$

for $n=2,3, \cdots$ and all real $\alpha_{1}, \alpha_{2}, \cdots$.

Following the natural line of development, Kolmogoroff $[1 ; 2]$ was led to his celebrated necessary and sufficient conditions (the "three series theorem") for convergence of series,

$$
\sum_{1}^{\infty} f_{n}(t)
$$

An address delivered before the Annual Meeting of the Society in Columbus, Ohio, on December 28, 1948, by invitation of the Committee to Select Hour Speakers for Annual and Summer Meetings; received by the editors November 1, 1948.

${ }_{1}^{1}$ Numbers in brackets refer to the references cited at the end of the paper.

${ }^{2}$ Here, as in the sequel, $E\{\}$ denotes the set of $t$ 's satisfying the condition inside the braces, and $|A|$ denotes the Lebesgue measure of the set $A$. 
of arbitrary independent functions or, equivalently, series of independent random variables.

Simultaneously with the above development another theory was being evolved. In 1924 Kolmogoroff [3] proved that (1.3) also implies the convergence almost everywhere of the trigonometric gap series,

$$
\sum_{1}^{\infty} c_{k} \sin 2 \pi n_{k} t
$$

where $\left\{n_{k}\right\}$ is a sequence of integers satisfying the "Hadamard gap" condition

$$
\frac{n_{k+1}}{n_{k}}>q>1
$$

A few years later Zygmund [1] proved the counterpart of this result to the effect that (1.4) implies divergence almost everywhere of (1.7).

The analogies in behavior of Rademacher series (1.2) and trigonometric gap series (1.7) were so striking that considerable attention was devoted to the subject.

Paley and Zygmund $[1 ; 2 ; 3]$ discovered many other important analogies and discussed a variety of related topics while Banach [1] was led to a general study of so-called "lacunary series" which include as special cases both Rademacher and trigonometric gap series.

One important analogy, however, escaped investigation. Since the Rademacher functions are independent, it follows from the central limit theorem of the calculus of probability that

$$
\lim _{n \rightarrow \infty}\left|\underset{t}{E}\left\{\sum_{1}^{n} c_{k} r_{k}(t)<\omega\left(\sum_{1}^{n} c_{k}^{2}\right)^{1 / 2}\right\}\right|=(2 \pi)^{-1 / 2} \int_{-\infty}^{\omega} e^{-u^{2} / 2} d u
$$

provided

$$
\sum_{1}^{\infty} c_{k}^{2}=\infty
$$

and

$$
\max _{1 \leqq k \leqq n}\left|c_{k}\right|=o\left\{\left(\sum_{1}^{n} c_{k}^{2}\right)^{1 / 2}\right\}
$$

It was only natural to inquire whether a similar result holds if the $r_{k}(t)$ are replaced by $\sin 2 \pi n_{k} t$ or, more generally, by $f\left(n_{k} t\right)$. This problem was the starting point of a series of investigations which will be reviewed in Part $\mathrm{I}$. 
Part II will be devoted to a review of a class of results in analytic number theory which again have been strongly influenced by the central limit theorem and other probabilistic results.

The limitations of time prevent us from reviewing other fields (notably the theory of almost periodic functions) in which methods of probability theory were successfully employed.

\section{PART I. GAP SERIES}

2. The trigonometric cases. One of the oldest tools of probability theory is the method of moments. The following lemma was first used by Tchebysheff:

If a sequence of distribution functions $\sigma_{n}(u)$ is such that for $l=0,1,2, \ldots$

$$
\lim _{n \rightarrow \infty} \int_{-\infty}^{\infty} u^{l} d \sigma_{n}(u)=(2 \pi)^{-1 / 2} \int_{-\infty}^{\infty} u^{l} e^{-u^{2} / 2} d u
$$

then

$$
\lim _{n \rightarrow \infty} \sigma_{n}(\omega)=(2 \pi)^{-1 / 2} \int_{-\infty}^{\omega} e^{-u^{2} / 2} d u
$$

for every real $\omega$.

By a simple restatement of this result we obtain the following: If $f_{n}(t)$ is a sequence of measurable functions defined in $(0,1)$ and if for $l=1,2, \ldots$

$$
\lim _{n \rightarrow \infty} \int_{0}^{1} f_{n}^{l}(t) d t=(2 \pi)^{-1 / 2} \int_{-\infty}^{\infty} u^{l} e^{-u^{2} / 2} d u
$$

then

$$
\lim _{n \rightarrow \infty}\left|\underset{t}{E}\left\{f_{n}(t)<\omega\right\}\right|=(2 \pi)^{-1 / 2} \int_{-\infty}^{\omega} e^{-u^{2} / 2} d u .
$$

This suggests an attack on the problem of determining the limit of

$$
\left|\underset{t}{E}\left\{\frac{\sum_{1}^{n} c_{k} \sin 2 \pi n_{k} t}{\left(\sum_{1}^{n} c_{k}^{2}\right)^{1 / 2}}<\omega\right\}\right|,
$$

as $n \rightarrow \infty$.

If instead of the gap condition 


$$
n_{k+1} / n_{k}>q>1
$$

we have the stronger condition

$$
n_{k+1} / n_{k} \rightarrow \infty,
$$

then, letting

$$
f_{n}(t)=2^{1 / 2} \frac{\sum_{1}^{n} c_{k} \sin 2 \pi n_{k} t}{\left(\sum_{1}^{n} c_{k}^{2}\right)^{1 / 2}},
$$

it is quite easy to verify that (2.3) holds provided $c_{k}=O(1)$ or, more generally,

$$
\max _{1 \leqq k \leqq n}\left|c_{k}\right|=o\left\{\left(\sum_{1}^{n} c_{k}^{2}\right)^{1 / 2}\right\}
$$

and

$$
\sum_{1}^{\infty} c_{k}^{2}=\infty
$$

Thus under conditions (2.7), (2.8), and (2.9) (Kac [1])

$$
\begin{aligned}
\lim _{n \rightarrow \infty} \mid \underset{t}{E}\left\{\sum_{1}^{n} c_{k} \sin 2 \pi n_{k} t\right. & \left.<\omega\left(\frac{1}{2} \sum_{1}^{n} c_{k}^{2}\right)^{1 / 2}\right\} \mid \\
& =(2 \pi)^{-1 / 2} \int_{-\infty}^{\omega} e^{-u^{2} / 2} d u .
\end{aligned}
$$

The verification of (2.3) in the case of the gap condition (2.6) is more delicate. It was first done by Erdös in 1940, under the condition $c_{k}=O(1)$, but the proof was not published. It actually turns out that the proof can be carried out under the less restrictive condition $(2.8) .^{3}$ In 1947 Fortet and Ferrand [1] announced a proof of (2.10) under the gap condition (2.6) but failed to state any restrictions on the $c_{k}$ 's. This omission led Salem and Zygmund [1] to believe that the Fortet-Ferrand proof must have been incomplete. They then proceeded to give a proof of (2.10) using an adaptation of the method of characteristic functions rather than the method of moments. They also proved that (2.8) is a necessary condition for the validity of (2.10), thus making the analogy with sums $\sum_{1}^{n} c_{k} r_{k}(t)$ complete.

\footnotetext{
${ }^{3}$ This proof will appear in a paper by Erdös, Ferrand, Fortet and Kac.
} 
In a subsequent paper Salem and Zygmund [2] extended (2.10) to the case where the $n_{k}$ 's are not necessarily integers. This extension had also been announced by Fortet and Ferrand [1]. In this case (unlike the case of integral $n_{k}$ ) there is a definite advantage in using the Salem-Zygmund method because the moment method becomes rather tedious.

Among various extensions of the above results we mention one because we feel that it is of independent interest.

Let

$$
F(z)=\sum_{0}^{\infty} c_{k} z^{n_{k}}
$$

where

$$
\sum_{0}^{\infty}\left|c_{k}\right|^{2}=\infty, \quad \frac{n_{k+1}}{n_{k}}>q>1
$$

and

$$
\max _{1 \leqq k \leqq n}\left|c_{k}\right|=o\left\{\left(\sum_{0}^{n} c_{k}^{2}\right)^{1 / 2}\right\} .
$$

For $0<r<1$, set

$$
M(r)=\left(\frac{1}{2 \pi} \int_{0}^{2 \pi}\left|F\left(r e^{i \theta}\right)\right|^{2} d \theta\right)^{1 / 2}
$$

and consider the set $E_{r}(\Omega)$ of those $\theta$ for which

$$
\frac{F\left(r e^{i \theta}\right)}{M(r)} \in \Omega,
$$

where $\Omega$ is a measurable set.

Then

$$
\lim _{r \rightarrow 1}\left|E_{r}(\Omega)\right|=2 \iint_{\Omega} e^{-\left(u^{2}+v^{2}\right)} d u d v
$$

(Salem and Zygmund [2] and for an earlier weaker version Kac [1]).

3. The general case. In view of the results of the preceding section it has been conjectured that for sufficiently restricted periodic (with period 1) $f(t)$ satisfying the normalization condition

$$
\int_{0}^{1} f(t) d t=0
$$


and for integers $n_{k}$ satisfying (2.6) the limit

$$
\lim _{m \rightarrow \infty}\left|\underset{t}{E}\left\{\sum_{1}^{m} f\left(n_{k} t\right)<\omega m^{1 / 2}\right\}\right|
$$

is again given by a properly normalized Gaussian integral. (We first consider the simple case $c_{k}=1, k=1,2, \ldots$.)

It thus came as a surprise when simultaneously and independently of each other, Erdös and Fortet constructed an example showing that the limit (3.2) need not be Gaussian. The example ${ }^{4}$ is

$$
f(t)=\cos 2 \pi t+\cos 4 \pi t, \quad n_{k}=2^{k}-1,
$$

in which case the limit (3.2) can be shown to be

$$
\pi^{-1 / 2} \int_{0}^{1} \int_{-\infty}^{\omega / 2|\cos \pi x|} e^{-u^{2}} d u d x
$$

(Kac [2]).

More complicated examples can be constructed for which the limit (3.2) need not even exist.

The interest of the Erdös-Fortet example is enhanced by the fact that for $n_{k}=2^{k}$ one has the following general theorem (Fortet [1], $\mathrm{Kac}[\mathbf{3}])$ :

If $f(t)$ is periodic with period 1 and either satisfies a Lipschitz condition or is of bounded variation ${ }^{5}$ and if furthermore

$$
\int_{0}^{1} f(t) d t=0
$$

and

$$
\lim _{n \rightarrow \infty} \frac{1}{n} \int_{0}^{1}\left(\sum_{1}^{n} f\left(2^{k} t\right)\right)^{2} d t=\sigma^{2} \neq 0
$$

then

$$
\lim _{n \rightarrow \infty}\left|\underset{t}{E}\left\{\sum_{1}^{n} f\left(2^{k} t\right)<\omega n^{1 / 2}\right\}\right|=\frac{(2 \pi)^{-1 / 2}}{\sigma} \int_{-\infty}^{\omega} e^{-u^{2} / 2 \sigma^{2}} d u .
$$

Thus the arithmetic structure of the sequence $\left\{n_{k}\right\}$ is of relevance except in the case $f(t)=\sin 2 \pi t$ (or somewhat more generally $f(t)$ $=a \sin 2 \pi t+b \cos 2 \pi t)$. The result (3.7) clearly indicates that, in

${ }^{4}$ In Salem and Zygmund [2] this example is erroneously credited to Erdös alone.

5 These conditions can be slightly relaxed. See Kac [3]. It should be clear that here and henceforth $2^{k}$ can be replaced by $a^{k}$, where $a$ is an integer greater than 1 . 
general, the functions $f\left(2^{k} t\right)$ do not behave as if they were independent. In fact, if they did, one would expect

$$
\sigma^{2}=\int_{0}^{1} f^{2}(t) d t
$$

instead of (3.6). As Fortet [1] points out the dependence exhibited by $f\left(2^{n}\right) t$ is very much like that in a Markoff chain. In the treatment of $\mathrm{Kac}$ [3] the dependence of $f\left(2^{n} t\right)$ is also brought out but unfortunately the connection with Markoff chains is lost.

It was pointed out by Erdös (see the final remark in Kac [3]) that (3.7) can be extended as follows:

If

$$
\sum_{1}^{\infty} c_{k}^{2}=\infty, \quad c_{k}=O(1)
$$

and if

$$
\liminf _{n \rightarrow \infty} \frac{\int_{0}^{1}\left(\sum_{1}^{n} c_{k} f\left(2^{k} t\right)\right)^{2} d t}{\sum_{1}^{n} c_{k}^{2}}>0
$$

then

$$
\begin{aligned}
\lim _{n \rightarrow \infty} \mid \underset{t}{E}\left\{\sum_{1}^{n} c_{k} f\left(2^{k} t\right)\right. & \left.<\omega\left(\int_{0}^{1}\left(\sum_{1}^{n} c_{k} f\left(2^{k} t\right)\right)^{2} d t\right)^{1 / 2}\right\} \mid \\
& =(2 \pi)^{-1 / 2} \int_{-\infty}^{\omega} e^{-u^{2} / 2} d u .
\end{aligned}
$$

It may be worth mentioning that from (3.7) it is not difficult to conclude that

$$
\begin{aligned}
\lim _{n \rightarrow \infty} \mid \underset{t}{E}\left\{\sum_{1}^{n} f\left(\left(2^{k}-1\right) t\right)\right. & \left.<\omega n^{1 / 2}\right\} \mid \\
& =\frac{1}{\pi} \int_{0}^{1} \int_{-\infty}^{\omega / g(x)} e^{-u^{2}} d u d x,
\end{aligned}
$$

where $g(x)$ is defined as follows:

Assume for the sake of simplicity that $f(t)$ is even, so that

$$
f(t) \sim \sum_{1}^{\infty} a_{n} \cos 2 \pi n t
$$


$\left(a_{0}=0\right.$ in view of (3.5)) and set, for $s=0,1,2, \cdots$,

$$
\begin{aligned}
& \alpha_{s}(t)=\sum_{n=0}^{\infty} a_{(2 s+1) 2^{n}} \cos 2 \pi(2 s+1) 2^{n} t, \\
& \beta_{s}(t)=\sum_{n=0}^{\infty} a_{(2 s+1) 2^{n}} \sin 2 \pi(2 s+1) 2^{n} t,
\end{aligned}
$$

then

$$
g(x)=\left(\sum_{s=0}^{\infty}\left(\alpha_{s}^{2}(x)+\beta_{s}^{2}(x)\right)^{1 / 2} .\right.
$$

This, of course, includes (3.4) as a special case. It is somewhat curious to note that if $f(t)$ contains no even harmonics $\left(a_{n}=0, n\right.$ even) we get

$$
g(x)=\text { const. }=\left(2 \int_{0}^{1} f^{2}(t) d t\right)^{1 / 2}
$$

and the distribution function on the right of (3.11) is again normal. An extension of (3.11) to sums

$$
\sum_{1}^{n} c_{k} f\left(\left(2^{k}-1\right) t\right)
$$

does not appear to be easy.

In contradistinction to the complex situation which prevails in the case of Hadamard gaps $n_{k+1} / n_{k}>q>1$, the case of "big gaps"

$$
n_{k+1} / n_{k} \rightarrow \infty
$$

is relatively simple. In fact we have the following theorem:

If $f(t)$ (periodic with period 1) satisfies a Lipschitz condition or is of bounded variation and if (3.5), (2.8) and (3.14) hold, then

$$
\begin{aligned}
\lim _{m \rightarrow \infty} \mid E_{t}\left\{\sum_{1}^{m} c_{k} f\left(n_{k} t\right)\right. & \left.<\omega\left(\sum_{1}^{n} c_{k}^{2}\right)^{1 / 2}\right\} \mid \\
& =\frac{(2 \pi)^{-1 / 2}}{\sigma} \int_{-\infty}^{\omega} e^{-u^{2} / 2 \sigma^{2}} d u,
\end{aligned}
$$

where

$$
\sigma^{2}=\int_{0}^{1} f^{2}(t) d t
$$

The proof follows from the following two facts: 
1. The theorem holds for a finite trigonometric polynomial

$$
S_{l}(t)=\sum_{r=1}^{l} a_{r} \cos 2 \pi r t
$$

2. For every $\epsilon>0$ one can choose an $S_{l}(t)$ (namely, a sufficiently high partial sum of the Fourier series of $f(t)$ ) in such a way that

$$
\int_{0}^{1}\left(\sum_{1}^{m} c_{k} f\left(n_{k} t\right)-\sum_{1}^{m} c_{k} S_{l}\left(n_{k} t\right)\right)^{2} d t<\epsilon \sum_{1}^{m} c_{k}^{2}
$$

for all $m$.

The first part can be proved by the method of moments and only slight modifications of the procedure used in $\mathrm{Kac}$ [1] are required. The proof of the second part parallels proofs of analogous statements in $\mathrm{Kac}[3]$ and [4]. for

It should be mentioned that Salem and Zygmund [2] showed that

$$
f(t)=\sum_{r=i}^{l} a_{r} \cos 2 \pi r t
$$

the conclusion (3.15) holds with (3.14) replaced by

$$
n_{k+1} / n_{k} \geqq q>l / j \text {. }
$$

In view of the Erdös-Fortet example this condition is best possible. The smoothness conditions imposed on $f(t)$ are needed in the proof of (3.18). ${ }^{6}$ The result (3.15) renders obsolete a weaker result in $\S 5$ of Kac [3].

4. Convergence and divergence of gap series. From (3.15) it follows almost immediately that

$$
\sum_{1}^{\infty} c_{k} f\left(n_{k} t\right)
$$

diverges almost everywhere provided

$$
\sum_{1}^{\infty} c_{k}^{2}=\infty
$$

For the Hadamard gaps the situation again becomes complex. We note first that, in general, divergence of $\sum c_{k}^{2}$ does not imply divergence almost everywhere of (4.1). In fact ( $\mathrm{Kac}[4])$, setting

' In proving (3.7) and (3.11) one also needs approximation theorems of type (3.18). Thus the necessity of imposing smoothness conditions on $f(t)$. 


$$
f(t)=g(t)-g(2 t), \quad n_{k}=2^{k}, \quad c_{k}=\frac{1}{k^{1 / 2}},
$$

we see that (4.2) is satisfied while the series (4.1) converges for every t.

However, for the case $n_{k}=2^{k}$, we can appeal to (3.10) and obtain the following:

If (4.2), $c_{k}=O(1)$ and, in addition,

$$
\liminf _{n \rightarrow \infty} \frac{\int_{0}^{1}\left(\sum_{1}^{n} c_{k} f\left(2^{k} t\right)\right)^{2} d t}{\sum_{1}^{n} c_{k}^{2}}>0
$$

then

$$
\sum_{1}^{\infty} c_{k} f\left(2^{k} t\right)
$$

diverges almost everywhere.

It is seen that in the example (4.3) the condition (4.4) is violated.

It is worth pointing out that the full strength of (3.10) is not really needed. It is sufficient to know that

(4.6) $\lim _{n \rightarrow \infty} \frac{\int_{0}^{1}\left(\sum_{1}^{n} c_{k} f\left(2^{k} t\right)\right)^{4} d t}{\left(\int_{0}^{1}\left(\sum_{1}^{n} c_{k} f\left(2^{k} t\right)\right)^{2} d t\right)^{2}}=3=(2 \pi)^{-1 / 2} \int_{-\infty}^{\infty} u^{4} e^{-u^{2} / 2} d u$.

In fact, setting

$$
F_{n}(t)=\frac{\sum_{1}^{n} c_{k} f\left(2^{k} t\right)}{\left(\int_{0}^{1}\left(\sum_{1}^{n} c_{k} f\left(2^{k} t\right)\right)^{2} d t\right)^{1 / 2}}
$$

we have

$$
\int_{0}^{1} F_{n}^{2}(t) d t=1, \quad \lim _{n \rightarrow \infty} \int_{0}^{1} F_{n}^{4}(t) d t=3
$$

and it is easy to show that $F_{n}(t)$ cannot approach 0 almost everywhere. ${ }^{7}$ Consequently (4.4) cannot converge almost everywhere and

\footnotetext{
${ }^{7}$ In fact, the measure of the set on which $F_{n}(t)$ converges to 0 cannot exceed $2 / 3$.
} 
hence must diverge almost everywhere. That the series (4.4) must converge or diverge almost everywhere is due to the fact that the gap sequence is $2^{k}$. For general gap sequences this may not be true.

It is an interesting open problem to decide whether the series

$$
\sum_{1}^{\infty} c_{k} f\left(n_{k} t\right), \quad \frac{n_{k+1}}{n_{k}}>q>1,
$$

can converge (diverge) on a set of positive measure without converging (diverging) almost everywhere.

With the exception of the case $n_{k}=2^{k}$, the whole question of divergence of series (4.1) for Hadamard gaps is also open.

In the trigonometric case, divergence of (4.2) implies divergence almost everywhere of

$$
\sum_{1}^{\infty} c_{k} \sin 2 \pi n_{k} t
$$

even in the case when the $n_{k}$ 's are not integers. This follows from (2.10) which can be regarded as a natural generalization of the result of Zygmund [1] and its extension to the non-harmonic case (Kac [5], Hartman [1]).

As far as convergence is concerned the situation is somewhat more satisfactory. We have, in fact, the following theorem (Kac [4]):

If

$$
\begin{gathered}
f(t+1)=f(t), \\
\int_{0}^{1} f(t) d t=0,
\end{gathered}
$$

and

$$
\left|f\left(t^{\prime}\right)-f\left(t^{\prime \prime}\right)\right| \leqq M\left|t^{\prime}-t^{\prime \prime}\right|^{\alpha}
$$$$
0<\alpha \leqq 1,
$$

then

$$
\sum_{1}^{\infty} c_{k} f\left(n_{k} t\right), \quad \frac{n_{k+1}}{n_{k}}>q>1,
$$

converges almost everywhere provided

$$
\sum_{1}^{\infty} c_{k}^{2}<\infty
$$

In contrast with previous results we are unable here to relax condition (4.9). However, at the expense of further restrictions on the sequence 
$\left\{n_{k}\right\}$, condition (4.9) can be considerably weakened. More specifically, we have the following:

If $f(t)$ satisfies (4.7), (4.8) and if for some $\alpha, 0<\alpha \leqq 1$,

$$
\int_{0}^{1}(f(t+h)-f(t))^{2} d t \leqq M h^{2 \alpha}
$$

and

$$
\sum_{1}^{\infty} 2^{-\alpha\left(m_{k}+1-m_{k}\right)}<\infty,
$$

then (4.11) implies convergence almost everywhere of the series

$$
\sum_{1}^{\infty} c_{k} f\left(2^{m k t}\right)
$$

( $m_{k}$ integers). (For a weaker result see Izumi and Kawata [1] and for a still weaker one $\mathrm{Kac}$ [6]). The interest of this theorem, in spite of its specialized character, lies in the fact that it can be deduced from Kolmogoroff's theorem concerning convergence of series of independent functions.

The proof is based on constructing a sequence of independent functions $g_{k}(t)$ such that

$$
\begin{gathered}
\int_{0}^{1} g_{k}(t) d t=0, \quad k=1,2, \cdots \\
\sum_{1}^{\infty} c_{k}^{2} \int_{0}^{1} g_{k}^{2}(t) d t<\infty
\end{gathered}
$$

and

$$
\sum_{1}^{\infty} \int_{0}^{1}\left|f\left(2^{m k} t\right)-g_{k}(t)\right| d t<\infty .
$$

That the existence of $g_{k}(t)$ implies our theorem is obvious since by Kolmogoroff's theorem (Kolmogoroff $[1 ; 2]$ ), (4.15) and (4.16) imply that

$$
\sum_{1}^{\infty} c_{k} g_{k}(t)
$$

converges almost everywhere and (4.17) implies (since $c_{k}=O(1)$ ) that

$$
\sum_{1}^{\infty} c_{k} g_{k}(t) \text { and } \sum_{1}^{\infty} c_{k} f\left(2^{m_{k} t}\right)
$$


are equiconvergent almost everywhere.

To construct the $g_{k}(t)$ divide the interval $(0,1)$ into $2^{r}$ equal subintervals and set

$$
\begin{aligned}
& f_{r}(t)=2^{r} \int_{k 2^{-r}}^{(k+1) 2^{-r}} f(x) d x \\
& k 2^{-r} \leqq t<(k+1) 2^{-r} ; \quad k=0,1, \cdots, 2^{r}-1 .
\end{aligned}
$$

It is evident that

$$
\begin{aligned}
\int_{0}^{1}\left(f(t)-f_{r}(t)\right)^{2} d t & \\
& =\sum_{k=0}^{2^{r}-1} 2^{r} \frac{1}{2} \int_{k 2^{-}}^{(k+1) 2^{-r}} \int_{k 2^{-r}}^{(k+1) 2^{-r}}(f(t)-f(s))^{2} d t d s .
\end{aligned}
$$

By an elementary change of variables we have

$$
\begin{aligned}
\frac{1}{2} \int_{k 2^{-r}}^{(k+1) 2^{-r}} \int_{k 2^{-r}}^{(k+1) 2^{-r}} & (f(t)-f(s))^{2} d t d s \\
& =\int_{0}^{2^{-r}} \int_{k 2^{-r}}^{(k+1) 2^{-r}-h}(f(t+h)-f(t))^{2} d t d h \\
& \leqq \int_{0}^{2^{-r}} \int_{k 2-r}^{(k+1) 2^{-r}}(f(t+h)-f(t))^{2} d t d h
\end{aligned}
$$

and consequently

$$
\begin{aligned}
\int_{0}^{1}\left(f(t)-f_{r}(t)\right)^{2} d t & \leqq 2^{r} \int_{0}^{2^{-r}} \int_{0}^{1}(f(t+h)-f(t))^{2} d t d h \\
& \leqq \frac{M}{2 \alpha+1} 2^{-2 r \alpha}
\end{aligned}
$$

Finally,

$$
\begin{aligned}
\int_{0}^{1}\left|f\left(2^{m k} t\right)-f_{r}\left(2^{m k} t\right)\right| d t & =\int_{0}^{1}\left|f(t)-f_{r}(t)\right| d t \\
& \leqq\left(\frac{M}{2 \alpha+1}\right)^{1 / 2} 2^{-r \alpha}
\end{aligned}
$$

Setting

$$
g_{k}(t)=f_{m_{k+1}-m_{k}}\left(2^{m_{k}} t\right)
$$

it is easy to verify that the $g_{k}(t)$ are independent and satisfy (4.15), 
(4.16) and (4.17)

5. Further results concerning convergence of gap series. Let $f(t)$ satisfy the conditions (4.7) and (4.8), and in addition assume that

$$
\int_{0}^{1}\left(f(t)-S_{n}(t)\right)^{2} d t=O(\lg n)^{-\alpha}
$$

where $\alpha>0$ and $S_{n}(t)$ is the $n$th partial sum of the Fourier series of $f(t)$. It can then be shown (Kac, Salem and Zygmund [1]) that:

(a) If $\alpha>2$ the series

$$
\sum_{1}^{\infty} c_{k} f\left(n_{k} t\right), \quad \frac{n_{k+1}}{n_{k}}>q>1,
$$

converges almost everywhere, provided

$$
\sum_{1}^{\infty} c_{k}^{2} \log ^{2} k<\infty
$$

(b) If $\alpha \leqq 2$, the series

$$
\sum_{1}^{\infty} \frac{f\left(n_{k} t\right)}{k^{1-\delta}}, \quad \frac{n_{k+1}}{n_{k}}>q>1,
$$

converges almost everywhere, provided

$$
\delta<\alpha / 4 \text {. }
$$

The $n_{k}$ 's need not be integers.

It follows immediately that under condition (5.1)

$$
\lim _{m \rightarrow \infty} \frac{1}{m} \sum_{1}^{m} f\left(n_{k} t\right)=0
$$

almost everywhere.

Erdös [1] pointed out that (5.6) holds under the weaker condition

$$
\int_{0}^{1}\left(f(t)-S_{n}(t)\right)^{2} d t=O(\lg \lg n)^{-1-\epsilon}, \quad \epsilon>0 .
$$

What is, however, more interesting is that he was able to construct an $f(t) \in L^{2}$ and a gap sequence $\left\{n_{k}\right\}$ of integers for which

$$
\limsup _{m \rightarrow \infty} \frac{1}{m} \sum_{1}^{m} f\left(n_{k} t\right)=\infty \text {. }
$$

From (5.8) it follows a fortiori that 


$$
\sum_{1}^{\infty} \frac{f\left(n_{k} t\right)}{k}
$$

diverges almost everywhere.

In view of this example it is apparent that in convergence theorems of gap series some restrictions on the function $f(t)$ (like (4.9), (4.12), or (5.1)) are necessary.

Let us finally mention that, for $n_{k}=2^{k},(5.6)$ follows from the ergodic theorem (F. Riesz [1], Raikov [1]) for every $f \in L$. We have here another example of the extreme sensitivity of gap theorems to the arithmetic structure of the gap sequence.

It is an open question whether for $n_{k}=q^{k}$ and $q>1$ not an integer, (5.6) holds for all $f \in L$, or at least for all $f \in L^{2}$.

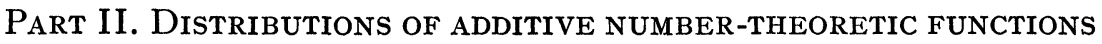

6. Preliminary remarks. A function $f(n), n=1,2, \cdots$, is called additive if

$$
f(m n)=f(m)+f(n)
$$

whenever

$$
(m, n)=1 \text {. }
$$

An additive function is thus completely determined if one knows

$$
f\left(p^{\alpha}\right), \quad p \text { a prime, } \alpha=1,2, \cdots .
$$

The function $f(n)$ is called strongly additive if

$$
f\left(p^{\alpha}\right)=f(p), \quad \alpha=2,3, \cdots .
$$

The simplest examples of strongly additive functions are

$$
\nu(n)=\text { number of prime divisors of } n
$$

(each prime divisor counted once) and

$$
\log \phi(n) / n
$$

where $\phi(n)$ is Euler's function.

In the first example $f(p)=1$ and in the second $f(p)=\log (1-1 / p)$. Let, for $p$ a prime,

$$
\rho_{p}(n)= \begin{cases}1, & p \mid n, \\ 0, & p \nmid n .\end{cases}
$$

With this notation the strongly additive function $f(n)$ can be written in the form 


$$
f(n)=\sum_{p} f(p) \rho_{p}(n)
$$

If $p_{1}, p_{2}, \cdots, p_{r}$ are distinct primes it follows trivially that

$$
\mathcal{D}\left\{\rho_{p_{1}}(n)=\epsilon_{1}, \cdots, \rho_{p_{r}}(n)=\epsilon_{r}\right\}=\prod_{k=1}^{r} \mathcal{D}\left\{\rho_{p_{k}}(n)=\epsilon_{k}\right\},
$$

where $\mathcal{D}\{\}$ denotes the density of integers which satisfy the condition inside the braces and each $\epsilon$ can be either 0 or 1. Formula (6.9) expresses the fact that the functions $\rho_{p}(n)$ are statistically independent and one might expect that the theory of addition of independent random variables can be applied to distribution problems of additive arithmetic functions.

Since the density is only a finitely additive measure, probability theorems will be directly applicable only if a finite number of $\rho_{p}$ 's is involved. Thus one can apply probability theorems to "truncated" functions

$$
f_{k}(n)=\sum_{p<t} f(p) \rho_{p}(n) .
$$

The passage from theorems concerning $f_{k}(n)$ to theorems concerning $f(n)$ must by necessity employ number theoretic arguments and it is these arguments that usually constitute the deeper parts of the proof. However, without the probabilistic connections it would have been extremely difficult even to guess some of the theorems.

7. The Hardy-Ramanujan theorem and its generalizations. In 1917 Hardy and Ramanujan [1] proved that almost every integer $m$ has approximately $\lg \lg m$ prime divisors.

The precise formulation is as follows: If $\psi(m) \rightarrow \infty$ (arbitrarily slowly) the density of integers for which

(7.1) $\lg \lg m-\psi(m)(\lg \lg m)^{1 / 2}<\nu(m)<\lg \lg m+\psi(m)(\lg \lg m)^{1 / 2}$ is 1 .

Due to the slowness with which $\lg \lg m$ increases we have the equivalent formulation: If $q_{n}$ is the number of integers $m, 1 \leqq m \leqq n$, for which

(7.2) $\lg \lg n-\psi(n)(\lg \lg n)^{1 / 2}<\nu(m)<\lg \lg n+\psi(n)(\lg \lg n)^{1 / 2}$, then

$$
q_{n} / n \rightarrow 1 \text {. }
$$

The original proof of this theorem was quite lengthy and was based on 
estimates of the number $\pi_{k}(n)$ of integers not greater than $n$ which have exactly $k$ prime divisors.

In 1936 Turán [1] gave an extremely simple and ingenious proof of (7.3) based on the easily verifiable relationship

$$
\lim _{n \rightarrow \infty} \frac{\sum_{m=1}^{n}(\nu(m)-\lg \lg n)^{2}}{n \lg \lg n}=1 .
$$

To get (7.3) one need only notice that

$$
\frac{\sum_{m=1}^{n}(\nu(m)-\lg \lg n)^{2}}{n \lg \lg n} \geqq \frac{\left(n-q_{n}\right) \psi^{2}(n)}{n} .
$$

One recognizes here immediately the familiar device of estimating probabilities of deviations from the second moment (Tchebysheff's inequality). A slight restatement of (7.4) is highly suggestive.

Let $k_{n}(\omega)$ be the number of integers $m, 1 \leqq m \leqq n$, for which

$$
\frac{\nu(m)-\lg \lg n}{(\lg \lg n)^{1 / 2}}<\omega .
$$

Set

$$
\sigma_{n}(\omega)=k_{n}(\omega) / n,
$$

note that $\sigma_{n}(\omega)$ is a distribution function and

$$
\frac{\sum_{1}^{n}(\nu(m)-\lg \lg n)^{2}}{n \lg \lg n}=\int_{-\infty}^{\infty} \omega^{2} d \sigma_{n}(\omega) .
$$

Formula (7.4) can now be written as

$$
\lim _{n \rightarrow \infty} \int_{-\infty}^{\infty} \omega^{2} d \sigma_{n}(\omega)=1
$$

and it also follows simply that

$$
\lim _{n \rightarrow \infty} \int_{-\infty}^{\infty} \omega d \sigma_{n}(\omega)=0 .
$$

Since

$$
\nu(m)=\sum_{p} \rho_{p}(m)
$$


is a sum of independent functions one might guess that, in some sense, the distribution of $\nu(m)$ will be normal. Formulas (7.8) and (7.9) suggest then that we might have

$$
\lim _{n \rightarrow \infty} \int_{-\infty}^{\infty} \omega^{k} d \sigma_{n}(\omega)=(2 \pi)^{-1 / 2} \int_{-\infty}^{\infty} \omega^{k} e^{-\omega^{2} / 2} d \omega
$$

for $k=3,4, \cdots$, in which case it would follow that

$$
\lim _{n \rightarrow \infty} \sigma_{n}(\omega)=\lim _{n \rightarrow \infty} \frac{k_{n}(\omega)}{n}=(2 \pi)^{-1 / 2} \int_{-\infty}^{\omega} e^{-u^{2} / 2} d u .
$$

Unfortunately it does not seem easy to carry out the proof along these lines because of the difficulties involved in calculating the higher moments

$$
\frac{\sum_{m=1}^{n}(\nu(m)-\lg \lg n)^{k}}{n(\lg \lg n)^{k / 2}} .
$$

We mention this approach because it is the most straightforward and, in our opinion, well worth pursuing.

A special case of (7.12), namely,

$$
\lim _{n \rightarrow \infty} \sigma_{n}(0)=1 / 2,
$$

was proved by Erdös [2] in 1937. This proof used a simplified version (due to Landau) of Brun's method. By a slight modification of Erdös' argument one can obtain (7.12) for every $\omega$ (LeVeque [1]). In fact, the only reason why Erdös did not prove (7.12) in its full generality seems to be that he was unaware of the formula

$$
\lim _{x \rightarrow \infty} e^{-x} \sum_{k<x+\omega x^{1 / 2}} \frac{x^{k}}{k !}=(2 \pi)^{-1 / 2} \int_{-\infty}^{\omega} e^{-u^{2} / 2} d u,
$$

except for the special case $\omega=0$.

It might be of interest to give a heuristic argument which indicates how (7.15) is related to the problem of the distribution of $\nu(m)$.

Denoting by $\pi_{k}(n)$ the number of integers not greater than $n$ having exactly $k$ prime divisors we have

$$
k_{n}(\omega)=\sum_{k<\lg \lg } \sum_{n+\omega(\lg \lg n)^{1 / 2}} \pi_{k}(n) .
$$

The classical result of Landau to the effect that 


$$
\pi_{k}(n) \sim \frac{n}{\lg n} \frac{(\lg \lg n)^{k-1}}{(k-1) !}
$$

makes it plausible that

$$
\frac{k_{n}(\omega)}{n} \sim \frac{1}{\lg n} \sum_{k<\lg \lg n+\omega(\lg \lg n)^{1 / 2}} \frac{(\lg \lg n)^{k-1}}{(k-1) !} .
$$

Now (7.12) follows from (7.15) by putting $x=\lg \lg n$.

The method of Erdös [2] was applicable only to $\nu(m)$ but in 1939 Erdös and $\mathrm{Kac}$ [1] proved a general theorem which also established a pattern of proof of rather wide applicability. The theorem in question is as follows:

Let

$$
f(m)=\sum_{p} f(p) \rho_{p}(m)
$$

be a strongly additive function and set

$$
\begin{aligned}
& A_{n}=\sum_{p<n} \frac{f(p)}{p}, \\
& B_{n}=\sum_{p<n} \frac{f^{2}(p)}{p} .
\end{aligned}
$$

Assume that

$$
B_{n} \rightarrow \infty, \quad f(p)=O(1),
$$

and denote by $k_{n}(\omega)$ the number of integers $m, 1 \leqq m \leqq n$, for which

$$
f(m)<A_{n}+\omega B_{n}^{1 / 2} ;
$$

then

$$
\lim _{n \rightarrow \infty} \frac{k_{n}(\omega)}{n}=(2 \pi)^{-1 / 2} \int_{-\infty}^{\omega} e^{-u^{2} / 2} d u .
$$

Considering first the truncated function

$$
f_{k}(m)=\sum_{p<k} f(p) \rho_{p}(m)
$$

one shows by a direct application of the central limit theorem that the density of integers $m$ for which

$$
f_{k}(m)<A_{k}+\omega B_{k}^{1 / 2}
$$


approaches, as $k \rightarrow \infty$, the normal integral

$$
(2 \pi)^{-1 / 2} \int_{-\infty}^{\omega} e^{-u^{2} / 2} d u
$$

This can be restated as follows:

If $l(n, k ; \omega)$ is the number of integers $m, 1 \leqq m \leqq n$, for which (7.25) holds, then

$$
\lim _{k \rightarrow \infty} \lim _{n \rightarrow \infty} \frac{l(n, k ; \omega)}{n}=(2 \pi)^{-1 / 2} \int_{-\infty}^{\omega} e^{-u^{2} / 2} d u .
$$

The crucial point is now to convert (7.26) into

$$
\lim _{n \rightarrow \infty} \frac{l\left(n, \alpha_{n}, \omega\right)}{n}=(2 \pi)^{-1 / 2} \int_{-\infty}^{\omega} e^{-u^{L} / 2} d u
$$

where $\alpha_{n} \rightarrow \infty$.

This can be done if

$$
\alpha_{n}=n^{\psi(n)}, \quad \psi(n) \rightarrow 0, \alpha_{n} \rightarrow \infty,
$$

but in the proof one needs a precise estimate of the number of integers not greater than $N$ not divisible by primes less than $K_{N}$ (in the limit as $N \rightarrow \infty$ faster than each fixed power of $K_{N}$ ). Such an estimate can be obtained by Brun's method. ${ }^{8}$ In contrast to Erdös [2] one needs here the full strength of this method. Finally, choosing $\psi(n)$ in such a way that

$$
1 / \psi(n)=o\left(B_{n}\right)
$$

and using the assumption $f(p)=O(1)$, one shows that the errors introduced by replacing $f(m), A_{n}, B_{n}$ by $f_{\alpha_{n}}(m), A_{\boldsymbol{\alpha}_{n}}, B_{\boldsymbol{\alpha}_{n}}$ are negligible and thus derives (7.24) from (7.27).

Let us also mention that from (7.12) one obtains by a very simple argument the following result:

If $d(m)$ denotes the number of divisors of $m$ and $r_{n}(\omega)$ the number of integers $m, 1 \leqq m \leqq n$, for which

$$
d(m)<2^{\lg \lg n+\omega(\lg \lg n) 1 / 2},
$$

then (Kac [7])

${ }^{8} \mathrm{~A}$ book devoted to Brun's method and its applications is being prepared by W. J. Harrington and J. B. Rosser. We also take this opportunity to correct a few minor omissions and misprints in Erdös and Kac [1]: (a) in the statement of Lemma 3 it should be added that the 0 -estimate is uniform in $i$; (b) in formula (iii) on p. 741 + should be replaced by - ; (c) in the last formula of $\$ 4$ (p. 742) $O(1)$ should be replaced by $o(1)$. 


$$
\lim _{n \rightarrow \infty} \frac{r_{n}(\omega)}{n}=(2 \pi)^{-1 / 2} \int_{-\infty}^{\omega} e^{-u^{2} / 2} d u .
$$

8. Further extensions. An important extension of (7.24) was stated without proof by Erdös [3]. His theorem is as follows:

Denoting by $k_{n}\left(\omega_{1}, \omega_{2}\right)$ the number of integers $m, 1 \leqq m \leqq n$, for which simultaneously

$$
f(m)<A_{n}+\omega_{1} B_{n}^{1 / 2}, \quad f(m+1)<A_{n}+\omega_{2} B_{n}^{1 / 2}
$$

we have under the assumptions (7.22)

$$
\lim _{n \rightarrow \infty} \frac{k_{n}\left(\omega_{1}, \omega_{2}\right)}{n}=\frac{1}{2 \pi} \int_{-\infty}^{\omega_{1}} \int_{-\infty}^{\omega_{2}} e^{-\left(u^{2}+v^{2}\right) / 2} d u d v \text {. }
$$

The intrinsic interest of this result (which can be extended to $f(m)$, $f(m+1), \cdots, f(m+r)$, for each fixed $r)$, and the fact that the method of proof is little known to number theorists made it seem worth while to supply a detailed proof. This was done by LeVeque [1] who also pointed out the following corollaries:

If $t_{n}(\omega)$ and $s_{n}(\omega)$ denote, respectively, the number of integers $m, 1$ $\leqq m \leqq n$, for which

$$
\begin{gathered}
\nu(m)<\nu(m+1)+\omega(2 \lg \lg n)^{1 / 2}, \\
d(m)<d(m+1) 2^{\omega(2 \lg \lg n)^{1 / 2}}
\end{gathered}
$$

then

$$
\lim _{n \rightarrow \infty} \frac{t_{n}(\omega)}{n}=\lim _{n \rightarrow \infty} \frac{s_{n}(\omega)}{n}=(2 \pi)^{-1 / 2} \int_{-\infty}^{\omega} e^{-u^{2} / 2} d u .
$$

In the particular case $\omega=0,(8.5)$ was proved by Erdös [4] in 1936.

The proof of (8.2) utilizes the fact that $f_{k}(m)$ and $f_{k}(m+1)$ are statistically independent (for every fixed $k$ ) and again makes use of Brun's method. The application of Brun's method is in this case considerably more tedious inasmuch as one needs the so-called two residue case.

Several other applications of the central limit theorem can be found in Erdös [3].

9. Kolmogoroff's "three series" theorem in the theory of additive functions. An important and interesting problem which received considerable attention in the literature was to decide under what conditions a strongly additive function 


$$
f(m)=\sum_{p} f(p) \rho_{p}(m)
$$

possesses a distribution function. We say that the distribution function $\sigma(\omega)$ is the distribution function of $f(m)$, if the density of integers for which $f(m)<\omega$ exists and is equal to $\sigma(\omega)$ at each continuity point of $\sigma$. It may also be noted that the case of general additive functions can be reduced to the case of strongly additive ones (Erdös [5]).

Generalizing a whole series of results (Schoenberg $[1 ; 2]$, Davenport [1], Erdös [5; 6]) Erdös [7] proved in 1938 that the convergence of the series

$$
\sum_{p} \frac{f^{*}(p)}{p} \text { and } \sum_{p} \frac{f^{* 2}(p)}{p}
$$

where

$$
f^{*}(p)= \begin{cases}f(p), & |f(p)| \leqq 1, \\ 1, & \mid f(p)>1,\end{cases}
$$

is a sufficient condition for the existence of the distribution function of $f(m)$. The result is best understood by bringing out its probabilistic nature (Erdös and Wintner [1]). If we consider the truncated function

$$
f_{k}(m)=\sum_{p<k} f(p) \rho_{p}(m)
$$

we see that it possesses a distribution function $\sigma_{k}(\omega)$ identical with the distribution function of

$$
S_{k}=\sum_{p<k} f(p) X_{p}
$$

where the $X_{p}$ 's are independent random variables such that

$$
\text { Prob. }\left\{X_{p}=0\right\}=1-1 / p, \quad \text { Prob. }\left\{X_{p}=1\right\}=1 / p .
$$

The Fourier-Stieltjes transform of $\sigma_{k}(\omega)$ is thus

$$
L_{k}(\xi)=\int_{-\infty}^{\infty} e^{i \xi \omega} d \sigma_{k}(\omega)=\prod_{p<k}\left(1-\frac{1}{p}+\frac{1}{p} e^{i \xi f(p)}\right) .
$$

By Kolmogoroff's three series theorem (Kolmogoroff $[1 ; 2]$ ) convergence of the series (9.2) implies convergence with probability 1 (and hence convergence in probability) of the sequence (9.5) and consequently the existence of a distribution function $\sigma(\omega)$ such that 


$$
L(\xi)=\int_{-\infty}^{\infty} e^{i \xi \omega} d \sigma(\omega)=\prod_{p}\left(1-\frac{1}{p}+\frac{1}{p} e^{i \xi f(p)}\right) .
$$

The distribution function $\sigma(\omega)$ is then the distribution function of the infinite series

$$
\sum_{p} f(p) X_{p}
$$

In the case of number theoretic functions, convergence, as $k \rightarrow \infty$, of $f_{k}(m)$ to $f(m)$ for every $m$ is trivial, but because density is only a finitely additive measure, one cannot draw the conclusion that $f(m)$ has a distribution function. Even if we knew that the distribution function of $f(m)$ exists it still would not follow that it must be the limit of $\sigma_{k}(\omega)$. It is, however, not difficult to show (Erdös [7, Lemma $1]$ ) that if the series (9.2) converge, $f_{k}(m)$ approaches $f(m)$ in measure, that is, the upper density of integers for which $\left|f(m)-f_{k}(m)\right| \geqq \epsilon$ approaches 0 , as $k \rightarrow \infty$, for every $\epsilon>0$. This, together with the fact that $\sigma_{k}(\omega) \rightarrow \sigma(\omega)$, implies that $\sigma(\omega)$ is indeed the distribution function of $f(m)$. Since, by Kolmogoroff's theorem, convergence of the series (9.2) is also a necessary condition for convergence of $\sigma_{k}(\omega)$ to $\sigma(\omega)$, it was natural to conjecture that it is also a necessary condition for the existence of the distribution function of $f(m)$. This indeed is the case (Erdös and Wintner [1]) although the proof is somewhat less elementary (it depends, for instance, on the result of Erdös and $\mathrm{Kac}[\mathbf{1}])$.

Finally, let us mention that from a general result of P. Lévy [1] it follows that $\sigma(\omega)$ is continuous if and only if the series

$$
\sum_{f(p) \neq 0} \frac{f(p)}{p}
$$

diverges. This was also proved by Erdös [7] by elementary number theoretic considerations.

S. BANACH

\section{REFERENCES}

1. Ueber einige Eigenschaften der lakunären trigonometrischen Reihen, Studia Mathematica vol. 2 (1930) pp. 207-220.

H. Davenport

1. Ueber Numeri Abundantes, Preuss. Akad. Wiss. Sitzungsber. (1930) pp. 830837.

P. ERDös

1. On the strong law of large numbers, Bull. Amer. Math. Soc. Abstract 54-5-191. The paper will appear in Trans. Amer. Math. Soc.

2. Note on the number of prime divisors of integers, J. London Math. Soc. vol. 12 (1937) pp. 308-314. 
3. On the distribution function of additive functions, Ann. of Math. vol. 47 (1946) pp. 1-20.

4. On a problem of Chowla and some related problems, Proc. Cambridge Philos. Soc. vol. 32 (1936) pp. 530-540.

5. On the density of some sequences of numbers I, J. London Math. Soc. vol. 10 (1935) pp. 120-125.

6. On the density of some sequences of numbers II, ibid. vol. 12 (1937) pp. 7-11.

7. On the density of some sequences of numbers III, ibid. vol. 13 (1938) pp. 119-127.

P. ERDös and M. KAC

1. The Gaussian law of errors in the theory of additive number theoretic functions, Amer. J. Math. vol. 62 (1940) pp. 738-742.

P. ERDös and A. WinTNER

1. Additive arithmetical functions and statistical independence, Amer. J. Math. vol. 61 (1939) pp. 713-721.

W. FELLER

1. The fundamental limit theorems in probability, Bull. Amer. Math. Soc. vol. 51 (1945) pp. 800-832.

J. FERRAND and R. ForTET

1. Sur des suites arithmétiques equiréparties, C. R. Acad. Sci. Paris vol. 224 (1947) pp. 514-515.

R. FORTET

1. Sur une suite également repartie, Studia Mathematica 9 (1940) pp. 54-69.

G. H. HARDY and S. RAMANUJAN

1. The normal number of prime factors of a number $n$, Quart. J. Math. Oxford Ser. vol. 48 (1917) pp. 76-92.

P. HARTMAN

1. The divergence of non-harmonic gap series, Duke Math. J. vol. 9 (1942) pp. 404405.

S. Izumi and T. Kawata

1. On certain series of functions, Tôhoku Math. J. vol. 46 (1939) pp. 91-105.

M. KAC

1. Note on power series with big gaps, Amer. J. Math. vol. 61 (1939) pp. 473-476.

2. Distribution properties of certain gap sequences, Bull. Amer. Math. Soc. Abstract 53-7-290. Details will be given in the paper by Erdös, Ferrand, Fortet and $\mathrm{Kac}$ (see footnote 2). The following misprint should be corrected: $\omega /|\cos \pi k|$ should be replaced by $\omega / 2|\cos \pi x|$.

3. On the distribution of values of sums of the type $\sum f\left(2^{k} t\right)$, Ann. of Math. vol. 47 (1946) pp. 33-49.

4. Convergence of certain gap series, ibid. 44 (1943) pp. 411-415.

5. Convergence and divergence of non harmonic gap series, Duke Math. J. vol. 8 (1941) pp. 541-545.

6. Sur les fonctions indépendantes V, Studia Mathematica vol. 7 (1938) pp. 96100.

7. Note on the distribution of values of the arithmetic function $d(m)$, Bull. Amer. Math. Soc. vol. 47 (1941) pp. 815-817.

M. KAC, R. SALEM and A. ZYGMUND

1. A gap theorem, Trans. Amer. Math. Soc. vol. 63 (1948) pp. 235-243.

S. KACZMARZ and H. STEInhaus

1. Le système orthogonal de M. Rademacher, Studia Mathematica vol. 2 (1930) pp. 231-247. 
A. Khintchine and A. KolmogorofF

1. Ueber Konvergenz von Reihen deren Glieder durch den Zufall bestimmt werden, Rec. Math. (Mat. Sbornik) vol. 32 (1925) pp. 668-677.

\section{A. KolMOGOROFF}

1. Ueber die Summen durch den Zufall bestimmter unabhängiger Grössen, Math. Ann. vol. 99 (1928) pp. 309-319.

2. Bemerkungen zu meiner Arbeit "Ueber die summen Zufälliger Grössen," ibid. vol. 102 (1929) pp. 484-489.

3. Une contribution a l'êtude de la convergence des séries de Fourier, Fund. Math. P. LEVY vol. 5 (1924) pp. 96-97.

1. Sur les sêries dont les termes sont des variables eventuelles indépendantes, Studia Mathematica vol. 3 (1931) pp. 119-155.

W. J. LEVEQUE

1. On the distribution of values of number-theoretic functions, Thesis, Cornell University.

R. E. A. C. Paley and A. Zygmund

1. On some series of functions (1), Proc. Cambridge Philos. Soc. vol. 26 (1930) pp. 337-357.

2. On some series of functions (2), ibid. pp. 458-474.

3. On some series of functions (3), ibid. vol. 28 (1932) pp. 190-205.

H. RADEMACHER .

1. Einige Sätze ueber Reihen von allgemeinenen Orthogonalfunktionen, Math. Ann.

D. RAIKov vol. 87 (1922) pp. 112-138.

1. On some arithmetical properties of summable functions, Rec. Math. (Mat. F. RIESz Sbornik) N.S. vol. 1 (1936) pp. 377-383.

1. Sur la théorie ergodique, Comment. Math. Helv. vol. 17 (1945) pp. 221-239.

R. SALEM and A. ZYGMUND

1. On lacunary trigonometric series, Proc. Nat. Acad. Sci. U.S.A. vol. 33 (1947) pp. 333-338.

2. On lacunary trigonometric series, ibid. vol. 34 (1948) pp. 54-62.

I. J. SCHOENBERG

1. Ueber die asymptötische Verteilung reeller Zahlen mod 1, Math. Zeit. vol. 28 (1928) pp. 171-200.

2. On asymptotic distribution of arithmetical functions, Trans. Amer. Math. Soc. vol. 39 (1936) pp. 315-330.

H. Steinhaus

1. Les probabilités dénombrables et leur rapport d la theorie de la mesure, Fund. Math. vol. 4 (1922) pp. 286-310.

P. TURÁN

1. Ueber einige Verallgemeinerungen eines Satzes von Hardy und Ramanujan, J. London Math. Soc. vol. 11 (1936) pp. 125-133.

A. ZYGMUND

1. On the convergence of lacunary trigonometric series, Fund. Math. vol. 16 (1930) pp. 90-107.

\section{Cornell University}

